\title{
Real-world data of six patients with atypical hemolytic uremic syndrome switched to ravulizumab
}

\author{
Rasmus Ehren $^{1}$ (D) Sandra Habbig ${ }^{1}$
}

Received: 3 May 2021 / Accepted: 3 May 2021 / Published online: 17 July 2021

(C) The Author(s) 2021

To the editors,

Ravulizumab, a long-acting C5-inhibitor, has been shown to be efficacious and safe in clinical studies in pediatric patients with preceding eculizumab treatment [1] and in therapy-naïve pediatric and adult patients $[2,3]$ with atypical hemolytic uremic syndrome (aHUS).

We present here the first real-world data of six pediatric patients with genetically proven aHUS switched to ravulizumab after a median time of 69 (range 6-123) months of eculizumab treatment. Four patients were diagnosed with a complement factor $\mathrm{H}(\mathrm{CFH})$ mutation, one patient with a complement factor 3 (C3) mutation, and one with DEAP-HUS (compare Supplementary Table 1 for detailed patient characteristics). Hematological and renal parameters remained stable as shown exemplarily for kidney function and lactate dehydrogenase (LDH) (Supplementary Figure 1). Comprehensive complement surveillance showed stable AP50 suppression and suppressed sC5b-9 levels 3 months after therapy switch as compared to before (Supplementary Figure 1). None of the patients reported any side effects of ravulizumab treatment in the current investigation interval of a median of 220 (range 90-274) days. Importantly, all patients reported a subjective benefit in quality of life due to the extended dosing interval.

Our data support the conclusion of Tanaka et al. [1] and add six definitely diagnosed patients with aHUS to the existing evidence that ravulizumab is effective and safe in pediatric patients. Thus, our data are a significant contribution to the growing body of evidence. In addition, we were able to show that the switch to ravulizumab is feasible in a real-life setting.

Rasmus Ehren

rasmus.ehren@uk-koeln.de

Pediatric Nephrology, Children's and Adolescents' Hospital, Department of Pediatrics, Faculty of Medicine and University Hospital Cologne, University of Cologne, Kerpener Str 62, 50937 Cologne, Germany
Supplementary Information The online version contains supplementary material available at https://doi.org/10.1007/s00467-021-05203-8.

Author contribution Not applicable.

Funding Open Access funding enabled and organized by Projekt DEAL.

Data Availability Not applicable.

Code availability Not applicable.

\section{Declarations}

Ethics approval Not applicable.

Consent to participate Not applicable.

Consent for publication Not applicable.

Conflict of interest Rasmus Ehren has no conflict of interest. Sandra Habbig has received honoraria for lecturing from Sanofi-Aventis Deutschland $\mathrm{GmbH}$ and honoraria for participation on Advisory Boards of Alexion Pharma GmbH and Greenovation Biotech GmbH.

Open Access This article is licensed under a Creative Commons Attribution 4.0 International License, which permits use, sharing, adaptation, distribution and reproduction in any medium or format, as long as you give appropriate credit to the original author(s) and the source, provide a link to the Creative Commons licence, and indicate if changes were made. The images or other third party material in this article are included in the article's Creative Commons licence, unless indicated otherwise in a credit line to the material. If material is not included in the article's Creative Commons licence and your intended use is not permitted by statutory regulation or exceeds the permitted use, you will need to obtain permission directly from the copyright holder. To view a copy of this licence, visit http://creativecommons.org/licenses/by/4.0/.

\section{References}

1. Tanaka K, Adams B, Aris AM, Fujita N, Ogawa M, Ortiz S, Vallee M, Greenbaum LA (2021) The long-acting C5 inhibitor, ravulizumab, is efficacious and safe in pediatric patients with atypical 
hemolytic uremic syndrome previously treated with eculizumab. Pediatr Nephrol 36:889-898

2. Ariceta G, Dixon BP, Kim SH, Kapur G, Mauch T, Ortiz S, Vallee M, Denker AE, Kang HG, Greenbaum LA, 312 Study Group (2021) The long-acting C5 inhibitor, ravulizumab, is effective and safe in pediatric patients with atypical hemolytic uremic syndrome naïve to complement inhibitor treatment. Kidney Int 100:225-237

3. Rondeau E, Scully M, Ariceta G, Barbour T, Cataland S, Heyne N, Miyakawa Y, Ortiz S, Swenson E, Vallee M, Yoon S-S, Kavanagh
D, Haller H, 311 Study Group (2020) The long-acting C5 inhibitor, Ravulizumab, is effective and safe in adult patients with atypical hemolytic uremic syndrome naïve to complement inhibitor treatment. Kidney Int 97:1287-1296

Publisher's note Springer Nature remains neutral with regard to jurisdictional claims in published maps and institutional affiliations. 